\title{
Quantitative study of geometrical scaling in deep inelastic scattering at HERA
}

\section{Michal Praszalowicz and Tomasz Stebel}

M. Smoluchowski Institute of Physics, Jagiellonian University, Reymonta 4, 30-059 Kraków, Poland

E-mail: michal@if.uj.edu.pl, tomasz.stebel@uj.edu.pl

ABSTRACT: We propose a method to assess the quality of geometrical scaling in Deep Inelastic Scattering and apply it to the combined HERA data on $\gamma^{*} p$ cross-section. Using two different approaches based on Bjorken $x$ binning and binning in $\gamma^{*} p$ scattering energy $W$, we show that geometrical scaling in variable $\tau \sim Q^{2} x^{\lambda}$ works well up to Bjorken $x$ 's 0.1 . The corresponding value of exponent $\lambda$ is $0.32-0.34$.

Keywords: Deep Inelastic Scattering (Phenomenology)

ArXiv EPRINT: 1211.5305 


\section{Contents}

1 Introduction 1

2 Bjorken $x$ binning $\quad 3$

$\begin{array}{llr}3 & \text { Energy binning } & 8\end{array}$

4 Summary and outlook 12

\section{Introduction}

Geometrical scaling (GS) introduced in ref. [1] in the context of low $x$ Deep Inelastic Scattering (DIS) is by now well established phenomenon attributed to the existence of an intermediate energy scale, called saturation scale $Q_{\mathrm{s}}(x)$. Saturation scale is defined as the border line between dense and dilute gluonic systems within a proton (for review see e.g. refs. $[2,3])$. GS has been also observed in DIS on nucleus [4-6] and in diffraction [7]. Recently it has been shown that GS is also exhibited by the $p_{\mathrm{T}}$ spectra at the LHC [813]. Geometrical scaling has a natural explanation within the theory of saturation and the Color Glass Condensate [14-19]. However, one should note that GS extends well above the saturation scale both in the DGLAP [20,21] and BFKL [22] evolution schemes. It has been argued in ref. [23] that the appearance of GS in course of the evolution is fairly independent of the initial conditions.

Geometrical scaling takes place if some observable that in principle depends on two independent kinematical variables like $Q^{2}$ (or $p_{\mathrm{T}}^{2}$ ) and $W$ (i.e. $\gamma^{*} p$ c.m.s. energy) depends only on a specific combination of them, namely on

$$
\tau=\frac{Q^{2}}{Q_{\mathrm{s}}^{2}(x)}
$$

called scaling variable. Here

$$
x=\frac{Q^{2}}{Q^{2}+W^{2}-M_{\mathrm{p}}^{2}}
$$

is Bjorken $x$ variable, $M_{p}$ stands for the proton mass, and the saturation scale $Q_{\mathrm{s}}$ takes the following form $[1,24,25]$

$$
Q_{\mathrm{s}}^{2}(x)=Q_{0}^{2}\left(\frac{x}{x_{0}}\right)^{-\lambda}
$$

Here $Q_{0}$ and $x_{0}$ are free parameters which can be fitted to the data within some specific model of DIS, and exponent $\lambda$ is a dynamical quantity of the order of $\lambda \sim 0.3$. Physical observable that exhibits GS in the case of DIS is $\gamma^{*} p$ cross-section $\sigma_{\gamma^{*} p}\left(x, Q^{2}\right)=$ $4 \pi^{2} \alpha_{\mathrm{em}} F_{2}\left(x, Q^{2}\right) / Q^{2}$. 
Although many authors, following the original paper by Stasto, Golec-Biernat and Kwecinski [1], have shown that GS is seen in DIS data, until now there was no quantitative, model independent analysis of its applicability domain. Different forms of scaling variable have been tested in a series of papers [26-29] where the so called Quality Factor $(\mathrm{QF})$ has been defined and used as a tool to assess the quality of geometrical scaling. These authors, however, constrained their analysis only to the domain of small Bjorken $x$ 's, $x<0.01$ and concentrated on testing the different forms of scaling variable $\tau$. The QF is a new tool for which the quantitative statements, like the one concerning the value of exponent $\lambda$ for example, are not based on the standard chi-square analysis.

In contrast, in this paper we propose a new numerical criterion for GS which serves as a tool to extract exponent $\lambda$ by standard $\chi^{2}$ minimization. We consider $\sigma_{\gamma^{*} p}\left(x_{i}, Q^{2}\right)$ for different fixed $x_{i}$ 's as functions of $Q^{2}$. Geometrical scaling hypothesis means that

$$
\sigma_{\gamma^{*} p}\left(x_{i}, Q^{2}\right)=\frac{1}{Q_{0}^{2}} F(\tau)
$$

where $F(\tau)$ is a universal dimensionless function of $\tau$. Therefore if cross-sections $\sigma_{\gamma^{*} p}\left(x_{i}, Q^{2}\right)$ for different $x_{i}$ 's are evaluated not in terms of $Q^{2}$ but in terms of $\tau$, they should fall on one universal curve (see figures 10 and 11 in section 4). This in turn means that if we calculate ratio of cross-sections for different Bjorken $x_{i}$ 's each expressed in terms of $\tau$, we should get unity (with an accuracy of a few percent) independently of $\tau$. This allows to determine power $\lambda$ by minimizing deviations of these ratios from unity. We can form as many ratios as there are different pairs of $x_{i}$ 's in overlapping regions of $Q^{2}$ (or more precisely of $\tau$ ). Needless to say that the best values of $\lambda$ 's extracted from different ratios should coincide within errors.

In an ideal case one would choose the lowest possible $x$ as the reference $x_{\text {ref }}$ to calculate the ratios of the cross-sections. Unfortunately there is a strong correlation between Bjorken $x$ 's and values of $Q^{2}$ measured by HERA [30], and therefore there is no single value of $x_{i}$ which covers all available values of $Q^{2}$. It turns out that the coverage in space of $W$ (rather than $x$ ) and $Q^{2}$ is much broader. Therefore in what follows we also study the quality of GS for $\sigma_{\gamma^{*} p}\left(W_{i}, Q^{2}\right)$ in bins of $W$ although this requires "rebinning" of the data which are provided by HERA experiments only in bins of $\left(x, Q^{2}\right)$. There is, however, an advantage of such a procedure, as it is almost identical to the analysis applied to the $p_{\mathrm{T}}$ spectra in $p p$ collisions at the LHC [8, 9, 11-13].

Since our analysis is sensitive only to the variations of scaling variable $\tau$ with $x$ and not to the absolute value of $\tau$, we choose in the following (unless specified otherwise) $Q_{0}=1 \mathrm{GeV} / c$ and $x_{0}=1$, i.e.:

$$
\tau=Q^{2} x^{\lambda} .
$$

The absolute value of the saturation scale can be inferred only from some explicit model of DIS at low $x$. For the purpose of the subsequent analysis we define $\gamma^{*} p$ cross-section as

$$
\sigma_{\gamma^{*} p}\left(x, Q^{2}\right)=\frac{F_{2}\left(x, Q^{2}\right)}{Q^{2}}
$$

since all proportionality constants cancel out in the ratios of the cross-sections that are the main tool used to look for geometrical scaling in this paper. The corresponding 


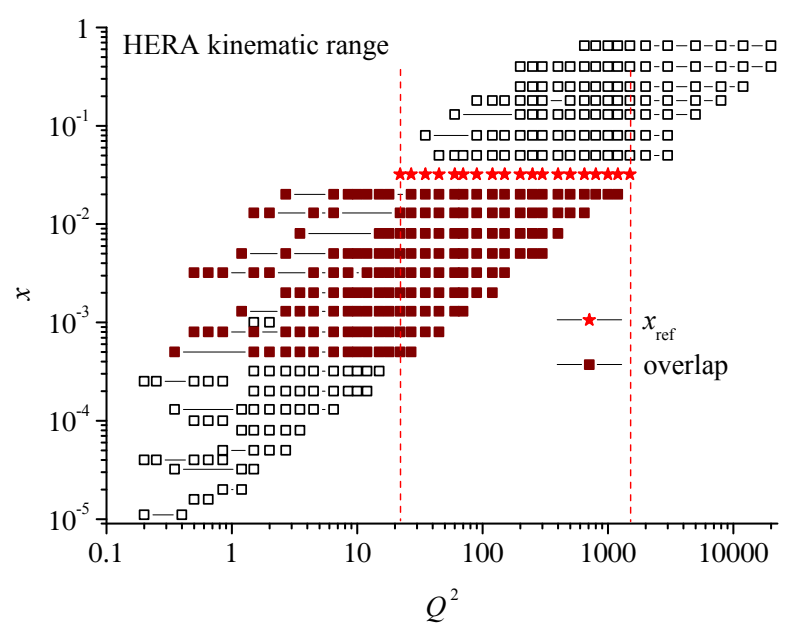

Figure 1. HERA kinematic range in $\left(Q^{2}, x\right)$ plane. It is shown which Bjorken $x$ 's (brown squares) are chosen for given $x_{\text {ref }}$ (red stars) to construct ratios of $\gamma^{*} p$ cross-sections in scaling variable $\tau$.

experimental error is therefore given by

$$
\Delta \sigma_{\gamma^{*} p}=\frac{\Delta F_{2}}{Q^{2}}
$$

where experimental error of $Q^{2}$ is essentially negligible. ${ }^{1}$

Our findings can be shortly summarized as follows. Geometrical scaling in variable (1.5) works well up to Bjorken $x$ 's 0.1. The corresponding value of exponent $\lambda$ is $0.32-0.34$.

The paper is essentially divided into two parts. In section 2 we discuss GS in terms of Bjorken variable $x$, whereas in section 3 in terms of $\gamma^{*} p$ scattering energy $W$, in both cases for $e^{+} p$ scattering. In section 4 we summarize our results and discuss other experiments and also HERA data for $e^{-} p$. We also present possible applications of the method proposed in this paper to test other possible forms of scaling variable $\tau$ and to investigate scaling of the charm cross-section. Preliminary results and technical details can be found in ref. [31].

\section{Bjorken $x$ binning}

Combined study of ZEUS and H1 [30] provides $432 e^{+} p$ data points for DIS structure function $F_{2}$ in terms of Bjorken $x$ and $Q^{2}$. Here we shall use 348 points with Bjorken $x$ 's that have at least 2 points in $Q^{2}$ [31]. This choice corresponds to $5.52 \times 10^{-6} \leq x \leq 0.65$. Since there is a strong correlation between $x$ and $Q^{2}$ as far as HERA kinematic range is concerned (see figure 1), the analysis outlined in section 1 requires some refinement. We apply here the following procedure. First we choose some $x_{\text {ref }}$ and consider all Bjorken $x_{i}$ 's smaller than $x_{\text {ref }}$ that have at least two overlapping points in $Q^{2}$ (or more precisely in

\footnotetext{
${ }^{1}$ We thank Halina Abramowicz and Iris Abt from ZEUS collaboration at HERA for clarification of this point.
} 
scaling variable $\tau$ ), as depicted in figure 1 (note, however, that when we make the same plot in terms of variable $\tau$ rather than $Q^{2}$ the overlaps change; the number of overlapping points gets smaller). We require that $x_{i}<x_{\text {ref }}$ because GS holds for small values of Bjorken $x$ and therefore by increasing $x_{\text {ref }}$ we will be able to see violations of GS. For $x_{i}<x_{\text {ref }}$ the ratios

$$
R_{x_{i}, x_{\mathrm{ref}}}\left(\lambda ; \tau_{k}\right)=\frac{\sigma_{\gamma^{*} p}\left(x_{i}, \tau\left(x_{i}, Q_{k}^{2} ; \lambda\right)\right)}{\sigma_{\gamma^{*} p}\left(x_{\mathrm{ref}}, \tau\left(x_{\mathrm{ref}}, Q_{k, \mathrm{ref}}^{2} ; \lambda\right)\right)} \text { with } \tau_{k}=\tau\left(x_{i}, Q_{k}^{2} ; \lambda\right)=\tau\left(x_{\mathrm{ref}}, Q_{k, \mathrm{ref}}^{2} ; \lambda\right)
$$

are greater than 1 for $\lambda=0$. By increasing $\lambda$ one shifts $R_{x_{i}, x_{\text {ref }}}\left(\lambda ; \tau_{k}\right)$ towards unity with an accuracy $\delta$ for all values of $\tau_{k}$ :

$$
R_{x_{i}, x_{\mathrm{ref}}}\left(\lambda ; \tau_{k}\right) \rightarrow 1 \pm \delta .
$$

Here $\delta$ stands for theoretical accuracy of GS hypothesis for which we take $3 \%$. GS is an approximate scaling law and with accurate combined HERA data we see its tiny violations which show up as an increase of ratios $\mathrm{R}$ with scaling variable $\tau$. Including small theoretical error makes our analysis immune to this effect.

Note that $\tau\left(x_{i}, Q_{k}^{2} ; \lambda=0\right)=Q_{k}^{2}$ and ratios (2.1) are essentially ratios of $\gamma^{*} p$ cross-sections at overlapping values of $Q_{k}^{2}$ (as in figure 1). For $\lambda \neq 0$ points of the same $Q^{2}$ but different $x$ 's correspond generally to different $\tau$ 's. In order to calculate ratios (2.1) at points $\tau_{k}$ corresponding to one fixed $x_{i}$, one has to interpolate the reference cross-section $\sigma_{\gamma^{*} p}\left(x_{\text {ref }}, \tau\left(x_{\text {ref }}, Q^{2} ; \lambda\right)\right)$ to $Q_{k, \text { ref }}^{2}$ such that $\tau\left(x_{\text {ref }}, Q_{k, \text { ref }}^{2} ; \lambda\right)=\tau_{k}$. Since $\gamma^{*} p$ cross-sections to a good accuracy lie on straight lines as functions of $\log Q^{2}$, we apply in the following the linear interpolation in $\log Q^{2}$. Interpolation errors are included in an overall error of $R_{x_{i}, x_{\text {ref }}}$ (see eq. (2.4) below).

It is clear than not all available Bjorken $x$ 's can be used as $x_{\text {ref }}$. If one chooses $x_{\text {ref }}$ too small then there are no $x<x_{\text {ref }}$ which have at least two points in $\tau$ (or $Q^{2}$ ) within $\sigma_{\gamma^{*} p}\left(\tau\left(x_{\text {ref }}, Q^{2}\right)\right)$ domain. It turns out that we can use only $x_{\text {ref }} \geq 3.2 \cdot 10^{-5}$.

In order to find optimal exponent $\lambda$ that minimizes deviations of ratios (2.1) from unity we form the chi-square measure

$$
\chi_{x_{i}, x_{\mathrm{ref}}}^{2}(\lambda)=\frac{1}{N_{x_{i}, x_{\mathrm{ref}}}-1} \sum_{k \in x_{i}} \frac{\left(R_{x_{i}, x_{\mathrm{ref}}}\left(\lambda ; \tau_{k}\right)-1\right)^{2}}{\Delta R_{x_{i}, x_{\mathrm{ref}}}\left(\lambda ; \tau_{k}\right)^{2}}
$$

where the sum over $k$ extends over all points of given $x_{i}$ that have overlap with $x_{\text {ref }}$. As already explained above, for $\lambda=0$ this is essentially the sum over all overlapping values of $Q_{k}^{2}$, for $\lambda \neq 0$ we choose measured points of $\sigma_{\gamma^{*} p}\left(\tau\left(x_{i}, Q_{k}^{2} ; \lambda\right)\right)$ and interpolate the reference cross-section in $Q^{2}$ to the point $Q_{k, \text { ref }}^{2}$ such that $\tau\left(x_{\text {ref }}, Q_{k, \text { ref }}^{2} ; \lambda\right)=\tau_{k}$.

Finally, the errors entering formula (2.3) are calculated using

$$
\begin{aligned}
& \Delta R_{x_{i}, x_{\mathrm{ref}}}\left(\lambda ; \tau_{k}\right)^{2}= \\
& =\left(\left(\frac{\Delta \sigma_{\gamma^{*} p}\left(x_{i}, \tau\left(x_{i}, Q_{k}^{2}\right)\right)}{\sigma_{\gamma^{*} p}\left(x_{i}, \tau\left(x_{i}, Q_{k}^{2}\right)\right)}\right)^{2}+\left(\frac{\Delta \sigma_{\gamma^{*} p}\left(x_{\mathrm{ref}}, \tau\left(x_{\mathrm{ref}}, Q_{k, \mathrm{ref}}^{2}\right)\right)}{\sigma_{\gamma^{*} p}\left(x_{\mathrm{ref}}, \tau\left(x_{\mathrm{ref}}, Q_{k, \mathrm{ref}}^{2}\right)\right)}\right)^{2}\right) R_{x_{i}, x_{\mathrm{ref}}}\left(\lambda ; \tau_{k}\right)^{2}+\delta^{2}
\end{aligned}
$$

where $\Delta \sigma_{\gamma^{*} p}\left(\tau\left(x, Q^{2}\right)\right)$ are experimental errors (or interpolated experimental errors with interpolation error included) of $\gamma^{*} p$ cross-sections (1.7). 

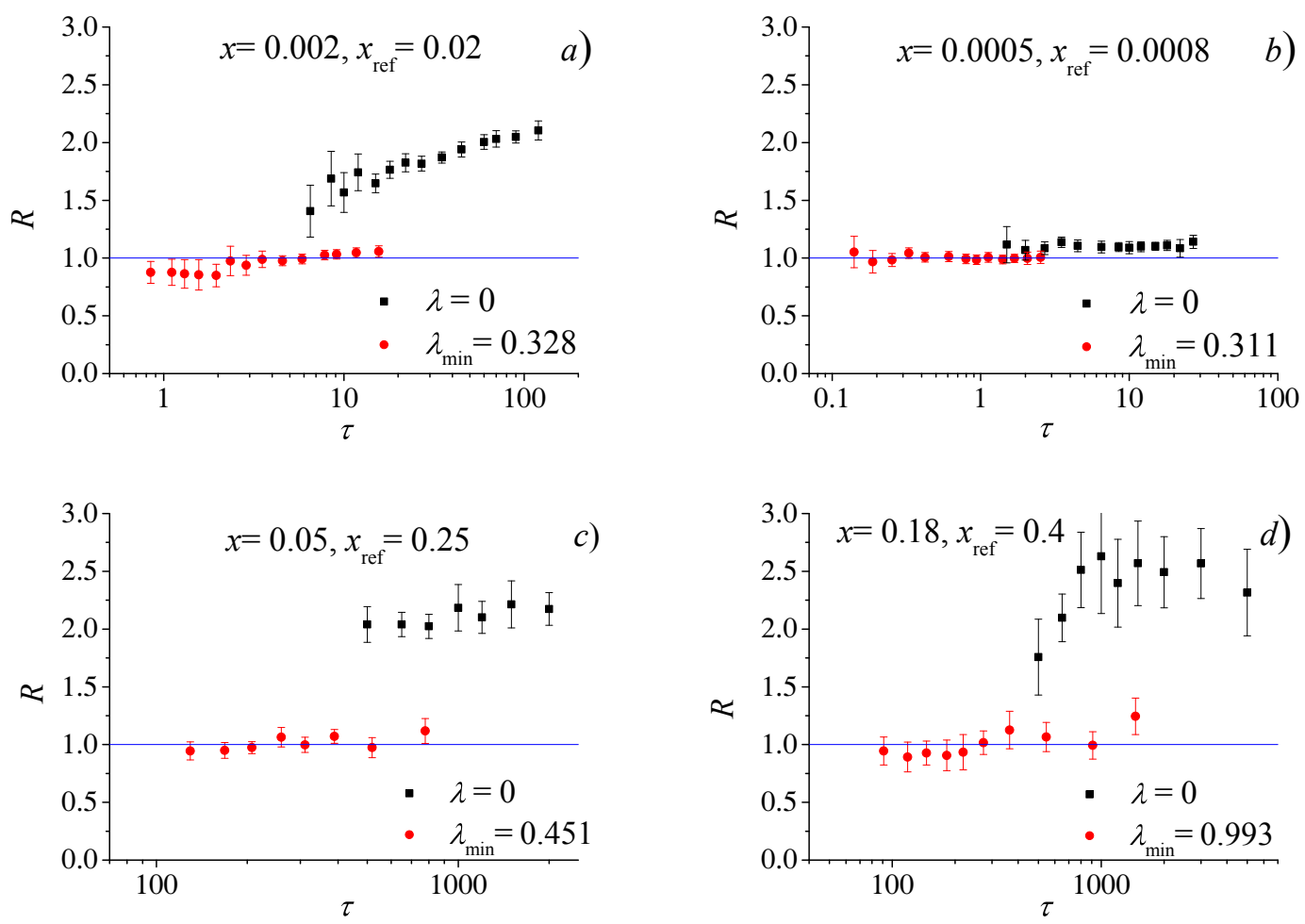

Figure 2. Ratios $R_{i}(\lambda)$ for $\lambda=0$ and $\lambda=\lambda_{\min }$ for some typical choices of $x$ and $x_{\text {ref }}$.

By minimizing $\chi_{x, x_{\text {ref }}}^{2}(\lambda)$ of eq. (2.3) with respect to $\lambda$ one can compute the "best" value $\lambda_{\min }\left(x, x_{\text {ref }}\right)$ and its error requiring that $\left(N_{x_{i}, x_{\text {ref }}}-1\right) \chi_{x_{i}, x_{\text {ref }}}^{2}(\lambda)$ changes by 1 around the minimum. In the region where GS is satisfied $\lambda_{\min }$ should not depend neither on $x$ nor on $x_{\text {ref }}$ and $\chi_{x, x_{\text {ref }}}^{2}\left(\lambda_{\min }\right)$ should be small. In figure 2 we plot ratios $R_{x, x_{\text {ref }}}\left(\lambda ; \tau_{k}\right)$ as functions of $\tau_{k}$ for $\lambda=0$ and $\lambda=\lambda_{\min }$ for some typical values of $x$ and $x_{\text {ref. }}$ In figure 2.a

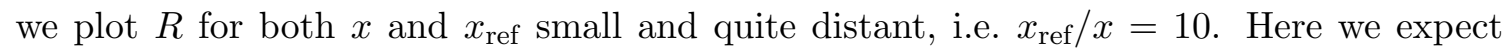
GS to be satisfied. We see that for $\lambda=0$ ratio $R$ grows with $\tau_{k}=Q_{k}^{2}$ and is of the order $1.5 \div 2$. By minimizing $\chi_{x, x_{\text {ref }}}^{2}(\lambda)$ we obtain $\lambda_{\min }=0.328$, which agrees with the expectations. In figure 2.b, both $x$ and $x_{\text {ref }}$ are small, however they are not very distant: $x_{\text {ref }} / x=1.6$. We see that already for $\lambda=0$ ratio $R_{x, x_{\text {ref }}}\left(0 ; \tau_{k}\right)$ is close to 1 ; we can improve the value of $\chi^{2}$ by increasing the value of $\lambda$ to $\lambda_{\min }=0.311$, in fair agreement with the previous case, however one should note that now $\chi^{2}(\lambda)$ is rather flat. Another example is shown in figure 2.c where $x_{\text {ref }}=0.25$ is relatively large and $x$ still quite small, such that $x_{\text {ref }} / x=5$. Here again it is possible to make $R_{x, x_{\text {ref }}}\left(\lambda ; \tau_{k}\right)$ close to unity, however at the expense of rather large value of $\lambda_{\min }=0.451$. This is a clear sign of violation of the universality of exponent $\lambda$, which we consider to be a signature of violation of geometrical scaling. Similarly in figure 2 .d we show $R_{x, x_{\text {ref }}}\left(\lambda ; \tau_{k}\right)$ for both $x$ and $x_{\text {ref }}$ large and not so close: $x_{\text {ref }} / x=2.22$. Here $\lambda_{\min }=0.993$, which is outrageously large. 


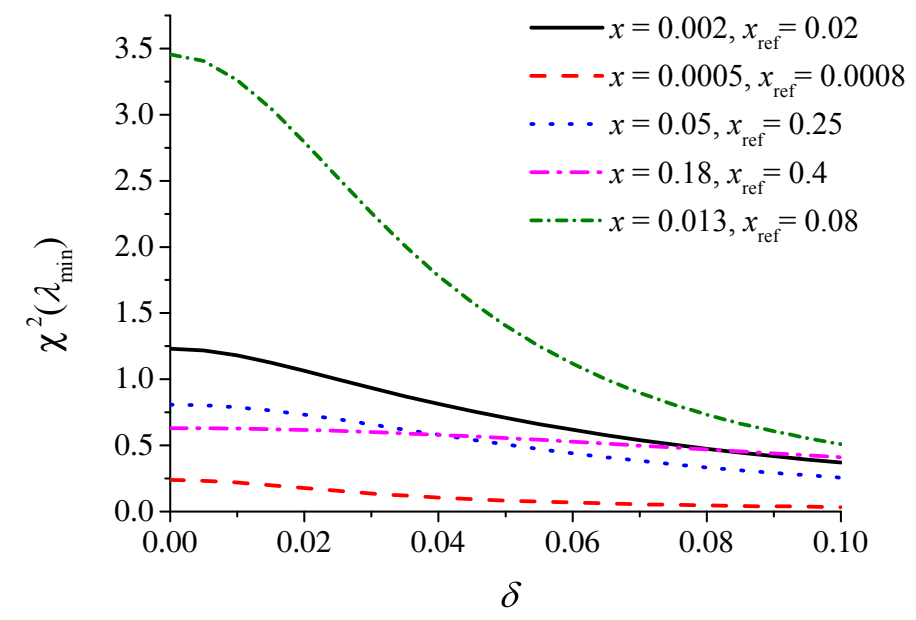

Figure 3. Values of $\chi^{2}\left(\lambda_{\min }\right)$ given by eq. (2.3) for four typical values of $x_{\text {ref }}$ and $x$ used in figure 2 and for the case with the largest $\chi^{2}$ (upper, green curve) as functions of theoretical error $\delta$.

Before we proceed, let us discuss the influence of small theoretical error $\delta$ introduced in eq. (2.1). To this end we plot in figure $3 \chi^{2}\left(\lambda_{\min }\right)$ given by eq. (2.3) for four typical values of $x_{\text {ref }}$ and $x$ used in figure 2 and for the case with the highest $\chi^{2}$ as functions of $\delta$. We see some moderate decrease of $\chi^{2}$ which even for $\delta=0$ are in most cases reasonably small. For the cases with high $\chi^{2}$, like the one represented by the upper curve in figure 3 , the decrease is a bit larger from 3.5 to 2.5 for $\delta=0.03$.

The qualitative measure of geometrical scaling is given by the independence of $\lambda_{\min }$ on Bjorken $x$ and by the value of $\chi_{x, x_{\text {ref }}}^{2}\left(\lambda_{\min }\right)$. In figure $4 \mathrm{w}$ show three dimensional plots of $\lambda_{\min }\left(x, x_{\mathrm{ref}}\right)$ and $\chi_{x, x_{\mathrm{ref}}}^{2}\left(\lambda_{\min }\right)$ in the $\left(x, x_{\mathrm{ref}}\right)$ plane. By construction both $\lambda_{\min }$ and $\chi^{2}\left(\lambda_{\min }\right)$ are defined only above the line $x_{\text {ref }}=x$. We see from figure 4.a that the stability corner of $\lambda_{\text {min }}$ (i.e. the region where variations of $\lambda_{\min }$ are small ) extends up to $x_{\text {ref }} \lesssim 0.1$. In the most of this region $0.3 \lesssim \lambda_{\min } \lesssim 0.4$. The value of $\chi_{x, x_{\text {ref }}}^{2}\left(\lambda_{\min }\right)$ displayed in figure 4 .b shows some fluctuations around unity except the region around $x_{\text {ref }} \sim 0.1$ (i.e. exactly where $\lambda_{\text {min }}$ starts growing) where it rises up to $\sim 2.2$. From figure 4 we can conclude that geometrical scaling holds up to Bjorken $x$ 's of the order of $10^{-1}$ which is well above the original expectations.

We can now look for possible violation of GS in more quantitative way. In order to eliminate the dependence of $\lambda_{\min }\left(x, x_{\text {ref }}\right)$ on the value of $x$, we introduce averages over $x$ (denoted in the following by $\langle\ldots\rangle$ ) minimizing the following chi-square function:

$$
\tilde{\chi}_{x_{\text {ref }}}^{2}(\langle\lambda\rangle)=\frac{1}{N_{x_{\text {ref }}}-1} \sum_{x<x_{\text {ref }}} \frac{\left(\lambda_{\min }\left(x, x_{\text {ref }}\right)-\langle\lambda\rangle\right)^{2}}{\Delta \lambda_{\min }\left(x, x_{\text {ref }}\right)^{2}}
$$

which gives the "best" value of $\lambda$ denoted as $\left\langle\lambda_{\min }\left(x_{\mathrm{ref}}\right)\right\rangle$. The sum in (2.5) extends over all $x$ 's such that $\lambda_{\min }\left(x, x_{\text {ref }}\right.$ ) exists (see figure 1). $N_{x_{\text {ref }}}$ is the number of terms in (2.5). The results are plotted in figure 5 as black squares. Errors $\Delta\left\langle\lambda_{\min }\right\rangle$ are calculated from the requirement that $\left(N_{x_{\text {ref }}}-1\right) \tilde{\chi}_{x_{\text {ref }}}^{2}(\langle\lambda\rangle)$ changes by 1 . For some points one can see 

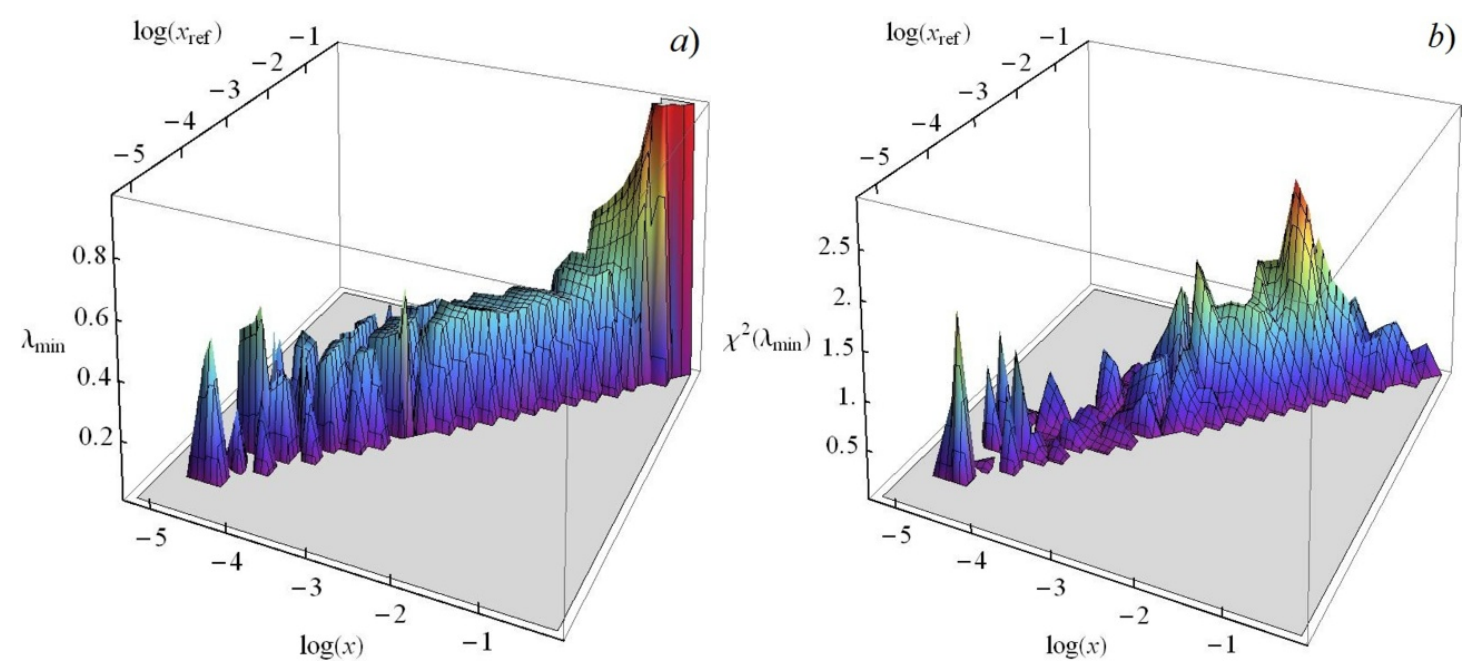

Figure 4. Three dimensional plots of a) $\lambda_{\min }\left(x, x_{\mathrm{ref}}\right)$ and b) the corresponding $\chi^{2}\left(\lambda_{\min }\right)$ given by eq. (2.3).
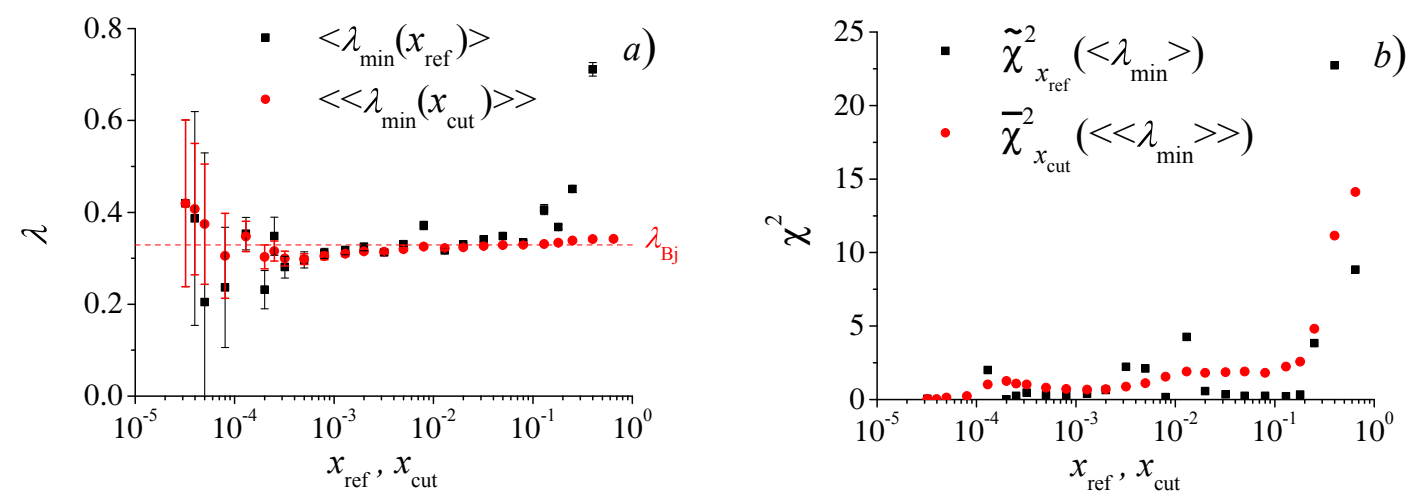

Figure 5. Left: averaged values $\left\langle\lambda_{\min }\left(x_{\mathrm{ref}}\right)\right\rangle$ (black squares) and $\left\langle\left\langle\lambda_{\min }\left(x_{\text {cut }}\right)\right\rangle\right\rangle$ (red circles); right: $\tilde{\chi}_{x_{\text {ref }}}^{2}$ (black squares) and $\bar{\chi}_{x_{\text {cut }}}^{2}$ (red circles) as functions of $x_{\text {ref }}$ and $x_{\text {cut }}$, respectively.

very large errors; this is due to the small number of possible $x$ 's for this particular $x_{\text {ref }}$. On average, however, one can approximate $\left\langle\lambda_{\min }\left(x_{\text {ref }}\right)\right\rangle$ with a constant value of $\sim 0.33$ up to $x_{\text {ref }} \sim 0.08$ where the rise of $\left\langle\lambda_{\min }\left(x_{\text {ref }}\right)\right\rangle$ should be interpreted as a violation of geometrical scaling. The corresponding $\tilde{\chi}_{x_{\text {ref }}}^{2}\left(\left\langle\lambda_{\text {min }}\right\rangle\right)$ is reasonably small up to $x_{\text {ref }}=0.2$ where it starts rapidly growing.

Looking at figure 5 one may have an impression that on the average $\left\langle\lambda_{\min }\left(x_{\mathrm{ref}}\right)\right\rangle$ is slightly rising with $x$. This small rise might be, however, attributed to the dependence of $\lambda$ on $Q^{2}$ through the kinematical correlation between Bjorken $x$ 's and $Q^{2}$ in the kinematical HERA range (see figure 1). $Q^{2}$ dependence of $\lambda$ has been measured in DIS for very small $x$ 's $[32,33]$ and has been discussed in the context of the $p_{\mathrm{T}}$ spectra at the LHC [10]. It is theoretically motivated by the corrections due the DGLAP evolution [34, 35]. We shall come back to this possibility in section 4 . 
To quantify further the hypothesis of geometrical scaling with constant $\lambda$ we form yet another chi-square function

$$
\bar{\chi}_{x_{\text {cut }}}^{2}(\langle\langle\lambda\rangle\rangle)=\frac{1}{N_{x_{\text {cut }}}-1} \sum_{x_{\text {ref }} \leq x_{\text {cut }}} \sum_{x<x_{\text {ref }}} \frac{\left(\lambda_{\min }\left(x, x_{\mathrm{ref}}\right)-\langle\langle\lambda\rangle\rangle\right)^{2}}{\Delta \lambda_{\min }\left(x, x_{\text {ref }}\right)^{2}}
$$

which we minimize to obtain $\left\langle\left\langle\lambda_{\min }\left(x_{\text {cut }}\right)\right\rangle\right\rangle$.

The idea behind equation (2.6) is to see how well one can fit $\left\langle\lambda_{\min }\left(x_{\mathrm{ref}}\right)\right\rangle$ with a constant $\lambda$ up to $x_{\text {ref }}=x_{\text {cut }}$. Were there any strong violations of GS above some $x_{0}$, one should see a rise of $\left\langle\left\langle\lambda_{\min }\left(x_{\text {cut }}\right)\right\rangle\right\rangle$ once $x_{\text {cut }}$ becomes larger than $x_{0}$. As can be seen from figure 5 , where $\left\langle\left\langle\lambda_{\min }\left(x_{\text {cut }}\right)\right\rangle\right\rangle$ and the corresponding $\bar{\chi}_{x_{\text {cut }}}^{2}$ are plotted as red circles, no drastic change in $\left\langle\left\langle\lambda_{\min }\left(x_{\text {cut }}\right)\right\rangle\right\rangle$ can be seen, although the slow rise is seen above $x_{\text {cut }} \simeq 0.1$. On the contrary $\bar{\chi}_{x_{\text {cut }}}^{2}$ starts to rise slowly at $x_{\text {cut }}=0.08$ and then rises steeply above $x_{\text {cut }} \simeq 0.2$.

Summarizing discussion of figure 5 we conclude that the best value of a constant $\lambda$ corresponds to $x_{\text {cut }}=0.08$ which we denote as

$$
\lambda_{\mathrm{Bj}}=0.329 \pm 0.002
$$

where subscript "Bj" stands for Bjorken $x$ binning. The error is purey statistical, we shall discuss systematic uncertainties in the end of section 3 and in section 4 .

Let us finish by a remark on comparison of $\left\langle\left\langle\lambda_{\min }\left(x_{\text {cut }}\right)\right\rangle\right\rangle$ with a more "differential" quantity $\left\langle\lambda_{\min }\left(x_{\mathrm{ref}}\right)\right\rangle$ depicted in figure 5.a. Since $\left\langle\left\langle\lambda_{\min }\left(x_{\text {cut }}\right)\right\rangle\right\rangle$ is in a sense an average of all $\left\langle\lambda_{\min }\left(x_{\text {ref }}\right)\right\rangle$ for $x_{\text {ref }} \leq x_{\text {cut }}$, the rapid increase of $\left\langle\lambda_{\min }\left(x_{\text {ref }}\right)\right\rangle$ above $x_{\text {ref }} \simeq 0.08$ is smoothed out due to a long constant tail of $\left\langle\lambda_{\min }\left(x_{\mathrm{ref}}\right)\right\rangle$ for smaller $x_{\text {ref }}$ 's. Below $x=0.08$ we have that $\left\langle\lambda_{\min }(x)\right\rangle \simeq\left\langle\left\langle\lambda_{\min }(x)\right\rangle\right\rangle$ which further confirms validity of geometrical scaling in this region.

\section{$3 \quad$ Energy binning}

Here we are going to repeat analysis of section 2 for the combined HERA data [30], but in bins of $\left(W, Q^{2}\right)$ rather than $\left(x, Q^{2}\right)$. The reason is that fixed energy points span over much wider common $Q^{2}$ range (see figure 6) and therefore we do not have to choose different reference points and average over them. Moreover, analysis in terms of $W$ follows exactly the method used to analyze $p_{\mathrm{T}}$ spectra at the LHC $[8,9,11-13]$. The disadvantage consists in the fact that HERA points are not organized in the energy bins, therefore we have to "rebin" them using formula (1.2). This leads to the loss of statistics. Indeed, instead of 432 points (see section 2) we shall use here $347 e^{+} p$ data points. Furthermore some ambiguities arise, such as the choice of the bin size, the need to recalculate Bjorken $x$ and others discussed in detail in ref. [31]. In what follows we shall use logarithmic binning with step 1.3 - every consecutive $W$ is 1.3 times larger than preceding one (in ref. [31] different binnings have been also considered without any major change of the results). We define value of energy $W$ as the mean of two limiting values $W_{\min }^{\prime}$ and $W_{\max }^{\prime}$ between which it lies (see table 1 ).

In what follows we use $W_{\text {ref }}=206 \mathrm{GeV}$ because it gives the widest range of $\tau$ values and is one of the biggest energies that we have at our disposal (GS is expected to be present 


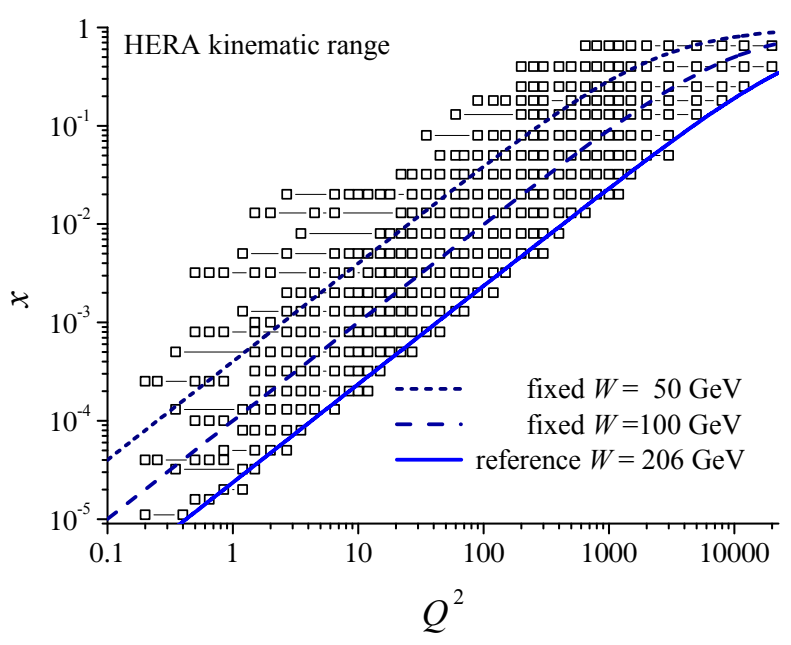

Figure 6. HERA kinematic range in $\left(Q^{2}, x\right)$ plane with several lines of constant $W$.

\begin{tabular}{|c|c|c|c|c|c|c|c|}
\hline$W_{\min }^{\prime}[\mathrm{GeV}]$ & 10 & 13 & 16.9 & 22 & 28.6 & 37.1 & 48.3 \\
\hline$W_{\max }^{\prime}[\mathrm{GeV}]$ & 13 & 16.9 & 22 & 28.6 & 37.1 & 48.3 & 62.7 \\
\hline$W[\mathrm{GeV}]$ & 11.5 & 15 & 19.4 & 25.3 & 32.8 & 42.7 & 55.5 \\
\hline Number of points & 6 & 3 & 13 & 22 & 22 & 32 & 33 \\
\hline$W_{\min }^{\prime}[\mathrm{GeV}]$ & 62.7 & 81.6 & 106 & 137.9 & 179.2 & 233 & \\
\hline$W_{\max }^{\prime}[\mathrm{GeV}]$ & 81.6 & 106 & 137.9 & 179.2 & 233 & 302.9 & \\
\hline$W[\mathrm{GeV}]$ & 72.2 & 93.8 & 122 & 158.5 & 206.1 & 267.9 & \\
\hline Number of points & 40 & 40 & 42 & 43 & 44 & 7 & \\
\hline
\end{tabular}

Table 1. Energy bins and energies assigned to them. Number of points in different bins is also displayed (these are values for $e^{+} p$ data).

at large energies), and it has the largest number of points in $Q^{2}$. In ref. [31] different choices of $W_{\text {ref }}$ have been also analyzed with essentially the same conclusions.

Similarly to the case of $x$-binning we shall construct ratios of $\gamma^{*} p$ cross-sections at different $W$ 's as functions of $\tau$. In general $\tau$ values for $W_{\text {ref }}$ and $W$ are different so we need to interpolate the reference cross-section to the value of $Q_{\text {ref }}^{2}$ which corresponds to the $\tau$ value needed to calculate the ratio. Here we follow closely the method of section 2 using linear interpolation in $\log Q^{2}$.

Now, for every point with energy $W_{i}$ we define a ratio ( $k$ labels points with energy $W_{i}$ but of different $\left.Q^{2}\right)$ :

$R_{W_{i}, W_{\mathrm{ref}}}\left(\lambda ; \tau_{k}\right):=\frac{\sigma_{\gamma^{*} p}\left(W_{\mathrm{ref}}, \tau\left(W_{\mathrm{ref}}, Q_{k, \mathrm{ref}}^{2} ; \lambda\right)\right)}{\sigma_{\gamma^{*} p}\left(W_{i}, \tau\left(W_{i}, Q_{k}^{2} ; \lambda\right)\right)}$ with $\tau_{k}=\tau\left(W_{i}, Q_{k}^{2} ; \lambda\right)=\tau\left(W_{\mathrm{ref}}, Q_{k, \mathrm{ref}}^{2} ; \lambda\right)$. 


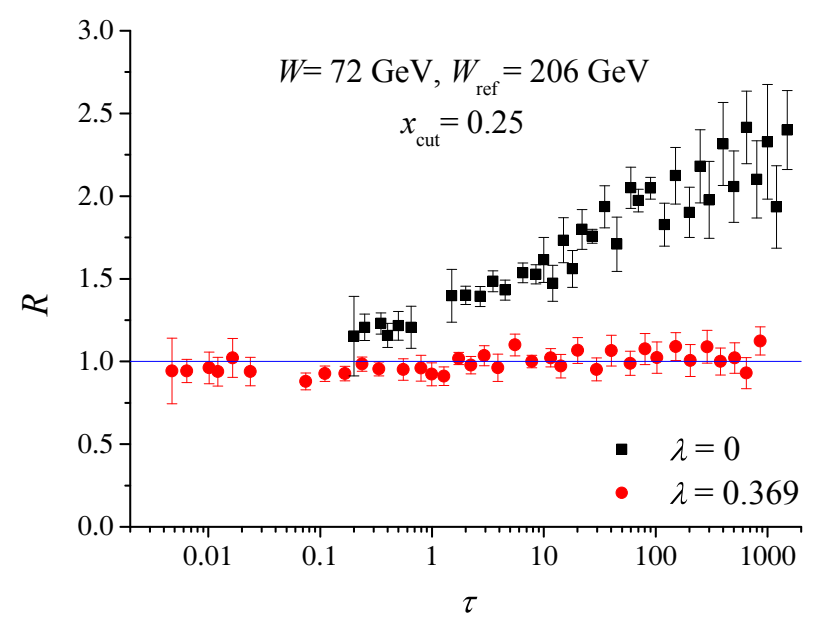

Figure 7. Ratios $R_{72}(\lambda)$ for $\lambda=0$ and $\lambda=\lambda_{\min }=0.369$ both with $x_{\text {cut }}=0.25$.

Uncertainty of ratio $R_{W_{i}, W_{\text {ref }}}\left(\lambda ; \tau_{k}\right)$ is given by:

$$
\begin{aligned}
& \Delta R_{W_{i}, W_{\text {ref }}}\left(\lambda ; \tau_{k}\right)^{2}= \\
& =\left(\left(\frac{\Delta \sigma_{\gamma^{*} p}\left(W_{i}, \tau\left(W_{i}, Q_{k}^{2}\right)\right)}{\sigma_{\gamma^{*} p}\left(W_{i}, \tau\left(W_{i}, Q_{k}^{2}\right)\right)}\right)^{2}+\left(\frac{\Delta \sigma_{\gamma^{*} p}\left(W_{\text {ref }}, \tau\left(W_{\text {ref }}, Q_{k, \text { ref }}^{2}\right)\right)}{\sigma_{\gamma^{*} p}\left(W_{\text {ref }}, \tau\left(W_{\text {ref }}, Q_{k, \text { ref }}^{2}\right)\right)}\right)^{2}\right) R_{W_{i}, W_{\text {ref }}}\left(\lambda ; \tau_{k}\right)^{2}+\delta^{2}
\end{aligned}
$$

Since we have fixed $W_{\text {ref }}=206 \mathrm{GeV}$, in what follows we shall omit subscript $W_{\text {ref. }}$.

In figure 7 we show as an example $R_{72}$ plotted for $\lambda=0$ and $\lambda=0.369$. We see that $R_{72}$ decreases several times when we use scaling variable rather than $Q^{2}$. This is generic feature which we employ to look for geometrical scaling.

Our aim is to find such $\lambda$ for given energy $W_{i}$ that deviations $R_{W_{i}}\left(\lambda ; \tau_{k}\right)-1$ are minimal. Taking into account uncertainties $\Delta R_{W}$ we define the chi-square function:

$$
\chi_{W_{i}}^{2}\left(x_{\mathrm{cut}} ; \lambda\right)=\frac{1}{N_{W_{i}, x_{\mathrm{cut}}}-1} \sum_{k \in W_{i} ; x \leq x_{\mathrm{cut}}} \frac{\left(R_{W_{i}}\left(\lambda ; \tau_{k}\right)-1\right)^{2}}{\Delta R_{W_{i}}\left(\lambda ; \tau_{k}\right)^{2}}
$$

where $k \in W_{i} ; x \leq x_{\text {cut }}$ means that we sum over points corresponding to given energy $W_{i}$ and values of $x$ that are not larger than $x_{\text {cut }}$. The reason to introduce a cut-off on Bjorken $x$ is to look for violations of GS once we get into the region of large $x$ 's.

We will search $\lambda_{\min }\left(W_{i}, x_{\text {cut }}\right)$ which minimizes $\chi_{W_{i}}^{2}$ for given $W_{i}$ and $x_{\text {cut }}$. Uncertainty of $\lambda_{\text {min }}$ is estimated by requiring that $\left(N_{W_{i}, x_{\mathrm{cut}}}-1\right) \chi_{W_{i}}^{2}\left(x_{\mathrm{cut}} ; \lambda\right)$ changes by 1 when $\lambda$ is varied around the minimum. The results for 12 different energy bins are plotted in figure 8 . One can see that in each $W_{i}$ bin $\lambda_{\min }\left(x_{\text {cut }}\right)$ can be approximated by a constant, although for lower energies a slight increase for $x_{\text {cut }} \geq 0.1$ is present. Large error bars for higher energies are due to the fact that $\chi_{W_{i}}^{2}$ gets flatter once $W_{i}$ is close to $W_{\text {ref. }}$ To conclude that GS is well satisfied we have to check whether the corresponding $\lambda_{\min }\left(W_{i}\right)$ for given $x_{\text {cut }}$ are energy independent. This condition is satisfied if we restrict the energy range to $W_{i} \geq 33 \mathrm{GeV}$. 

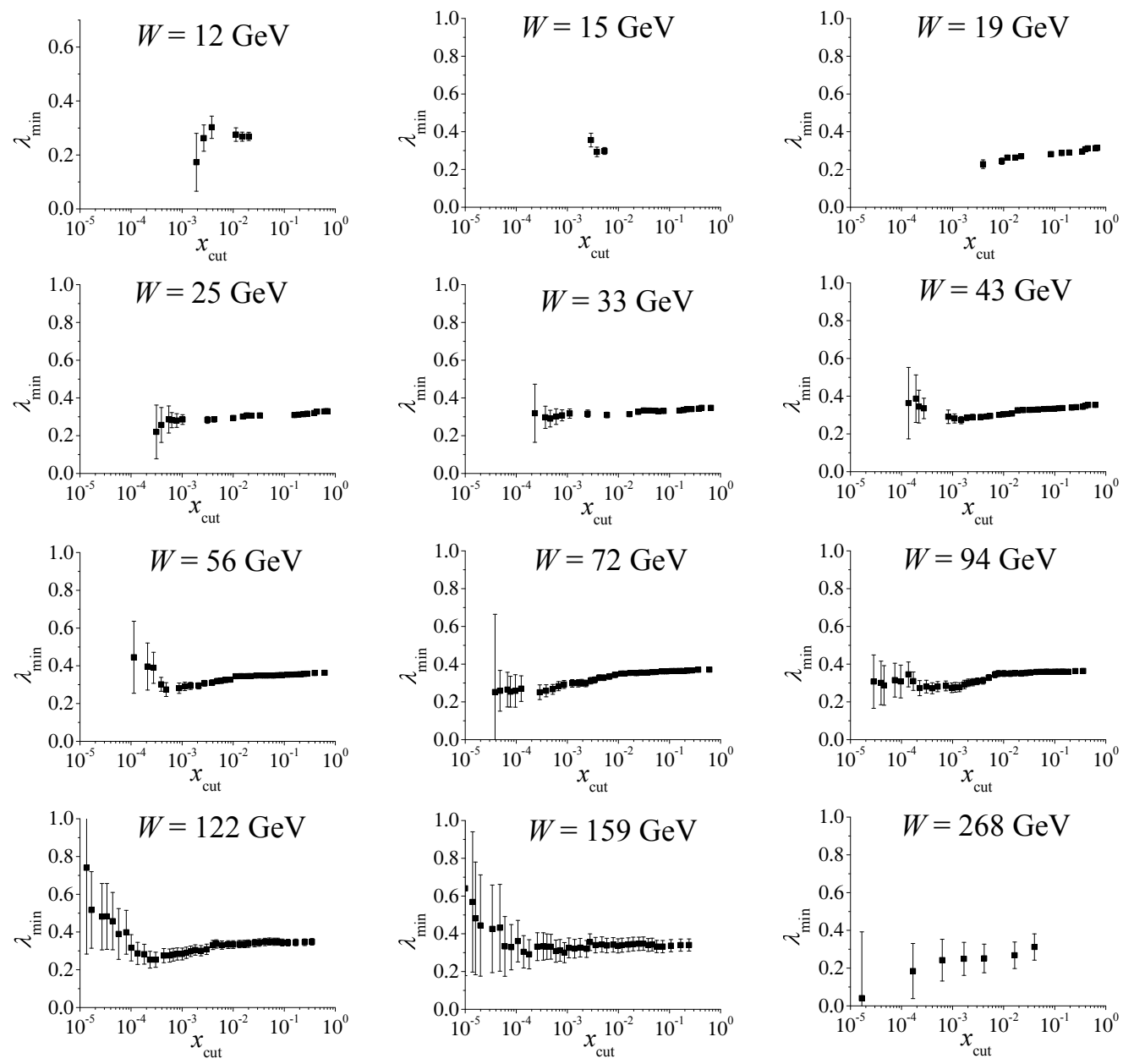

Figure 8. Exponent $\lambda_{\min }$ as a function of $x_{\text {cut }}$ for all energies $W \neq W_{\text {ref }}=206 \mathrm{GeV}$.

Therefore the final number of data points used in the analysis in this section is further reduced to 303. In figure 9.a we plot the corresponding values of $\chi_{W_{i}}^{2}$. One can see that up to $x_{\text {cut }} \simeq 0.1$ the $\chi_{W_{i}}^{2}$ 's are smaller than 1.2 , and the rapid growth is seen for $x_{\text {cut }}>0.1$.

In order to get rid of the energy dependence we define yet another chi-square function

$$
\tilde{\chi}^{2}\left(x_{\text {cut }} ; \lambda\right)=\frac{1}{N_{x_{\text {cut }}}-1} \sum_{i} \frac{\left(\lambda_{\min }\left(W_{i}, x_{\text {cut }}\right)-\lambda\right)^{2}}{\Delta \lambda_{\min }\left(W_{i}, x_{\text {cut }}\right)^{2}}
$$

where the sum goes over eight energies $W_{i}=33,43, \ldots, 159,268 \mathrm{GeV}$. By minimizing (3.4) with respect to $\lambda$ we obtain the best value of $\lambda_{\min }\left(W_{i}, x_{\text {cut }}\right)$ averaged over all energies denoted by $\lambda_{\text {ave }}\left(x_{\text {cut }}\right)$ which is plotted in figure $9 . \mathrm{b}$. The error of $\lambda_{\text {ave }}$ is calculated again by demanding that $\left(N_{x_{\text {cut }}}-1\right) \tilde{\chi}^{2}\left(x_{\text {cut }} ; \lambda\right)$ changes by 1 when $\lambda$ is varied around $\lambda_{\text {ave }}$. We see that $\lambda_{\text {ave }}\left(x_{\text {cut }}\right)$ is rather flat; large errors for small $x_{\text {cut }}$ are due to the small number of points with small $x$ 's. Looking at $\lambda_{\text {ave }}\left(x_{\text {cut }}\right)$ one does not see any dramatic change for $x_{\text {cut }} \rightarrow 1$. The values of $\tilde{\chi}^{2}\left(\lambda_{\text {ave }}\right)$ fluctuate around 1 with the highest value being smaller 2 for all $x_{\text {cut }}$ which is a clear sign of energy independence of $\lambda_{\min }$. 

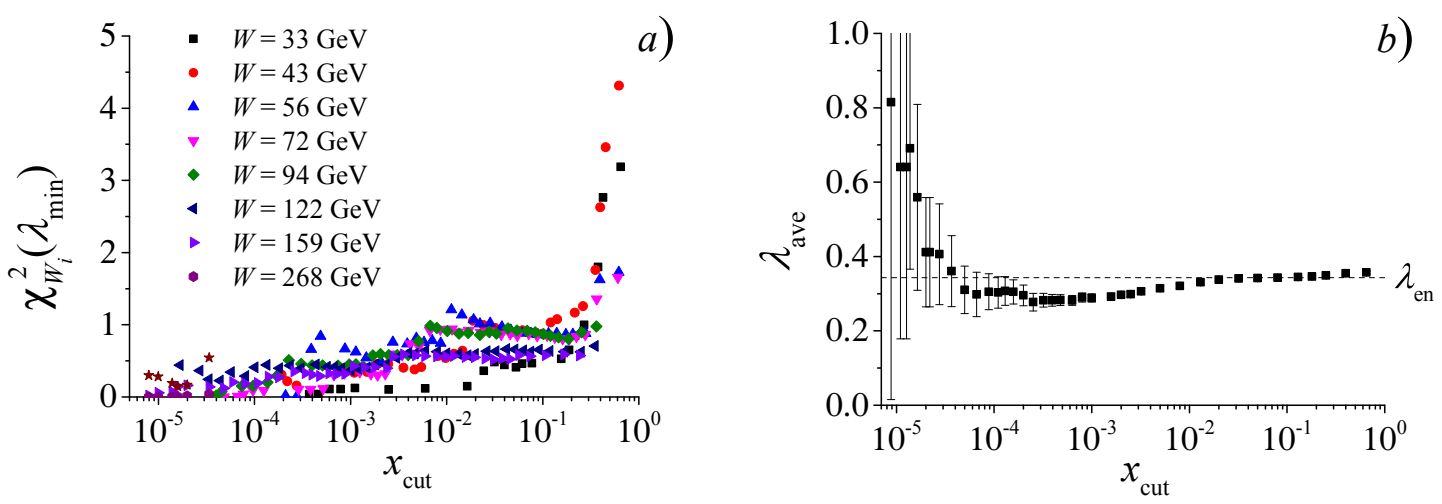

Figure 9. Left panel: plot of $\chi_{W_{i}}^{2}$ for different energies as functions of $x_{\text {cut }}$. Right panel: exponent $\lambda_{\min }$ averaged over energies (denoted as $\lambda_{\text {ave }}$ ) as a function of $x_{\text {cut }}$.

Summarizing, we conclude that for energy binning GS works well up to $x_{\text {cut }}=0.1$ where individual $\chi_{W_{i}}^{2}$ 's start growing, yelding

$$
\lambda_{\mathrm{en}}=\lambda_{\mathrm{ave}}\left(x_{\mathrm{cut}}=0.1\right)=0.343 \pm 0.004 .
$$

where subscript "en" stands for energy binning. The error here is purely statistical. Note, however, that $\lambda_{\mathrm{en}}$ is larger than $\lambda_{\mathrm{Bj}}$ and the difference is larger than the statistical errors. This difference may be used to asses accuracy of our method. Had we used all energies, not excluding $W_{i}$ 's up to $33 \mathrm{GeV}$, we would get $\lambda_{\text {en }}=0.329 \pm 0.003$, exactly as in the case of Bjorken binning.

\section{Summary and outlook}

In the present paper we have performed quantitative, model independent analysis of the accuracy and the applicability domain of geometrical scaling in deep inelastic $e^{+} p$ scattering. To this end we have chosen the most recent compilation of the HERA data based on common analysis of ZEUS and H1 experiments [30]. We have tested the standard form of geometrical scaling [1,24, 25] given by the form of the scaling variable $\tau$ (1.5), assuming the constant value of the exponent $\lambda$. In order to quantify the quality of GS we have proposed two different approaches. In the first approach we have computed ratios of cross-sections $F_{2} / Q^{2}$ for two different Bjorken $x$ 's, denoted as $x$ and $x_{\text {ref }}$, as functions of the scaling variable $\tau$; such ratios should be equal to unity if GS is present. This allowed us to extract the best value of the exponent $\lambda$, which we called $\lambda_{\min }\left(x, x_{\text {ref }}\right)$. In the region where GS is present $\lambda_{\min }\left(x, x_{\text {ref }}\right)$ should not depend on $x$ and $x_{\text {ref }}$, and moreover the corresponding $\chi^{2}$ should not be too large. In the second approach we have repeated essentially the same calculation but for $\gamma^{*} p$ cross-sections treated as functions of the scattering energy $W$ rather than $x$. This procedure is analogous to the one applied to the $p_{\mathrm{T}}$ spectra at the LHC. It requires, however, "rebinning" of the data, which reduces the statistics and introduces uncontrollable errors, for example the uncertainty of $x$, and also in principle some uncontrollable dependence on the choice of $W$ bins. Nevertheless, 

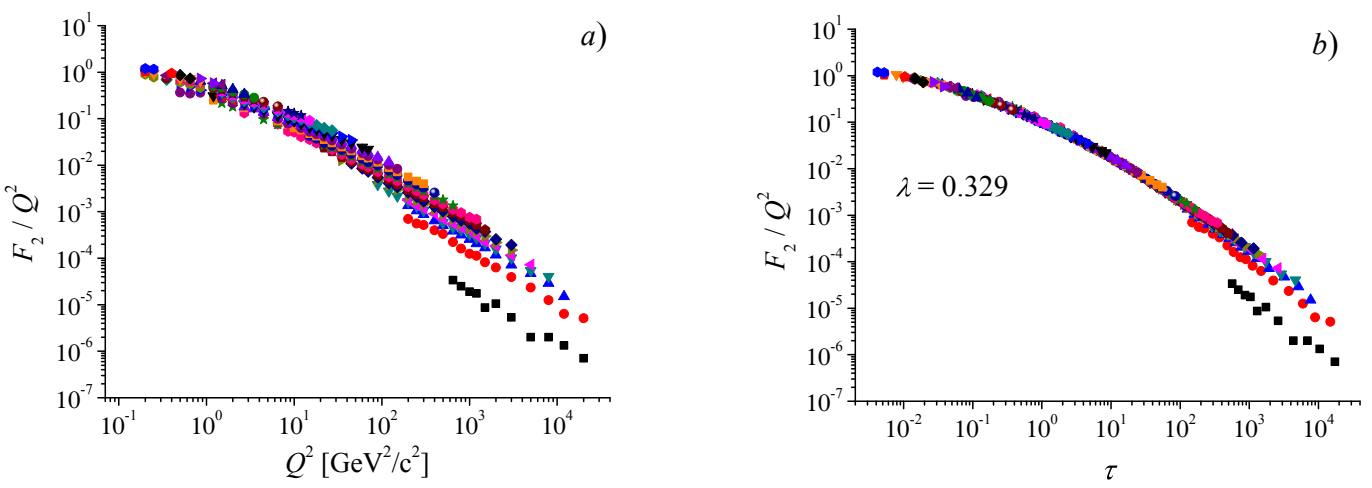

Figure 10. Geometrical scaling for the Bjorken binning. Left: $\gamma^{*} p$ cross-sections $F_{2} / Q^{2}$ as functions of $Q^{2}$ for fixed $x$. Different points correspond to different Bjorken $x$ 's. Right: the same but in function of scaling variable $\tau$ for $\lambda=0.329$. Points in the right end of the plot correspond to large $x$ 's (due to kinematical correlation of the HERA phase space), and therefore show explicitly violation of geometrical scaling.
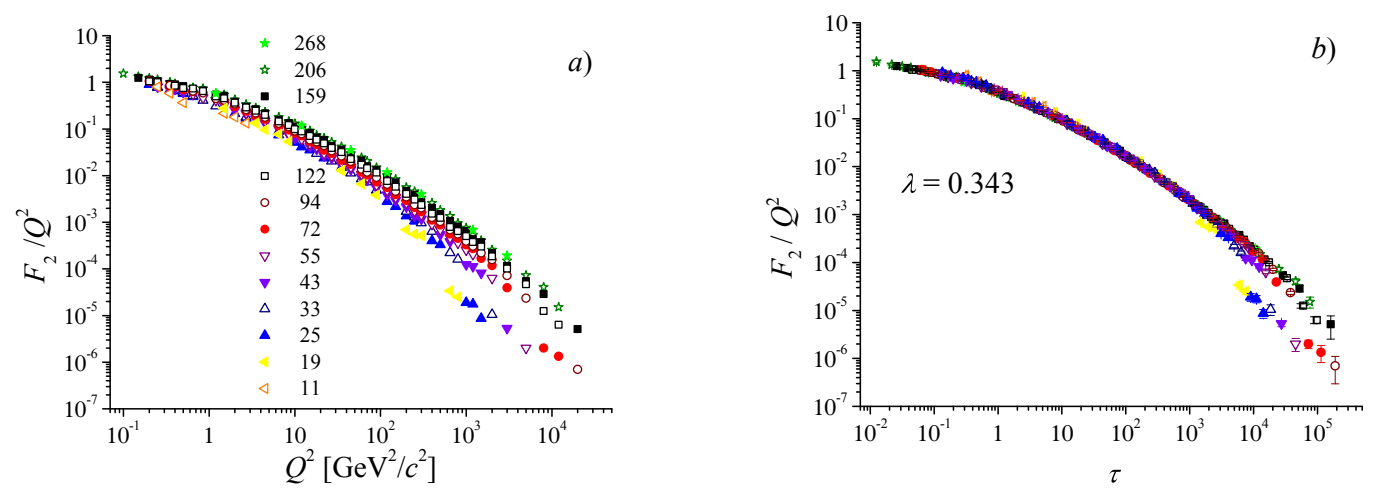

Figure 11. Geometrical scaling for the energy binning. Left: $\gamma^{*} p$ cross-sections $F_{2} / Q^{2}$ as functions of $Q^{2}$ for fixed $W$ 's. Right: the same but in function of scaling variable $\tau$ for $\lambda=0.343$. Points in the right end of the plot correspond to large $x$ 's and mostly small energies (due to kinematical correlation of the HERA phase space), and therefore show explicitly violation of geometrical scaling.

since $\gamma^{*} p$ cross-sections for all available energies overlap in $Q^{2}$ (or $\tau$ ) nearly over whole kinematical range (which is not the case for the $x$ binning, see figures 1 and 6 ), the analysis is much more straightforward than in the case of the Bjorken $x$ binning.

As mentioned in the Introduction we have assumed no error on $Q^{2}$, i.e. $\Delta Q^{2}=0$. In ref. [31] we have estimated $\Delta Q^{2}$ taking approximately half of the of the width of the $Q^{2}$ bin what should be considered as an upper bound of the real error. As a result the values of different $\chi^{2}$ functions discussed in that paper have been underestimated. However, since we never refer to an absolute value of any given $\chi^{2}$, but rather to the qualitative change of their behavior with $x_{\text {cut }}$ or $x_{\text {ref }}$, the conclusions concerning the validity of geometrical scaling do not depend much on actual values of $\Delta Q^{2}$ (with an obvious difference that theoretical 
error $\delta$ can be safely neglected once errors of $Q^{2}$ are large). Qualitative behavior of the $\chi^{2}$ functions is the same as in the present paper, although their values are smaller and their behavior with $x_{\text {cut }}$ or $x_{\text {ref }}$ more smooth. The values of $\lambda$, however, are essentially unchanged.

Despite the differences between the Bjorken $x$ binning and the energy binning the results in both cases are consistent and to some extent surprising. By averaging $\lambda_{\min }\left(x, x_{\mathrm{ref}}\right)$ or $\lambda_{\min }\left(W, W_{\text {ref }}\right)$ over the allowed regions of $x$ or $W$ respectively, and by inspecting the corresponding $\chi^{2}$ values, as described in detail in sections 2 and 3 , we have established that geometrical scaling holds up to relatively large $x$ 's of the order of 0.1 . The fact that GS works up to large Bjorken $x$ 's has been also observed in data analysis of ref. [36]. The resulting exponents $\lambda$ given by eqs. (2.7) and (3.5) are slightly different since in the latter case we removed points with $W \leq 25 \mathrm{GeV}$. Difference between those values shows systematic uncertainties arising from the change of binning. In any case exponents $\lambda$ obtained in this paper are consistent with previous estimates and model calculations, and read

$$
\lambda=0.32 \div 0.34
$$

These results are suggestively illustrated in figures 10 and 11 where we plot $\gamma^{*} p$ cross-sections for both types of binning used in this paper as functions of $Q^{2}$ and the scaling variable $\tau$ (with $Q_{0}=1 \mathrm{GeV} / c$ and $x_{0}=10^{-3}$ in eq. (1.3)). One can see that in both cases the cross-sections fall on one universal curve - a clear sign of geometrical scaling. Some deviations of GS can be seen in the lower right end of the plots. These points, however, correspond to large $x$ 's (due to the kinematical correlation of the HERA phase space, see figures 1,6), and therefore should not exhibit geometrical scaling.

The analysis performed here is essentially identical to the one presented in in ref. [31] with two important differences. Firstly, in ref. [31] we have used large errors of $Q^{2}$. Secondly, here, when interpolating $\gamma^{*} p$ cross-section in $Q^{2}$ we use linear interpolation in $\log Q^{2}$, rather than in $Q^{2}$ used in ref. [31]. The latter interpolation introduced large errors which were neglected in ref. [31]. This resulted in somewhat different values of the exponent $\lambda$. Qualitative picture, however, remains unchanged.

One of the questions addressed in [31] was possible dependence of $\lambda$ on $Q^{2}$. Applying the same method of dividing $\gamma^{*} p$ cross-sections of different Bjorken $x$ 's (or scattering energies $W$ ) we have been looking for the best exponent $\lambda$ which, in this case however, depended on $Q^{2}$. Instead of having one parameter $\lambda$ we had therefore a number of parameters $\lambda_{k}$ where $k$ runs over all $Q^{2}$ bins. By minimizing $\chi^{2}$ analogous to (2.3) or (3.3) we did not observe any visible dependence of $\lambda$ on $Q^{2}$. This is most probably due to the fact that the corresponding $\chi^{2}$ functions are very flat and one can easily fall into some accidental minimum. We therefore conclude that with the present experimental accuracy we have been unable to find $Q^{2}$ dependence of $\lambda$ in a model independent way. It seems that in this case one has to assume some Ansatz for $\lambda\left(Q^{2}\right)$ depending on a few variational parameters whose values can be found using the methods described in this paper. Universality of these parameters would then constitute a criterion for $Q^{2}$ dependence of $\lambda$. It is worthwhile to remark at this point that other variants of the saturation scale have been discussed in the literature (for discussion see e.g. [26, 27] and references therein). 
Application of our method to test the quality of GS for these different scaling variables (including $Q^{2}$ - dependent $\lambda$ ) is under preparation and will be presented elsewhere.

We have also analyzed separately $\gamma^{*} p$ cross-section for $e^{-} p$ scattering. The $e^{-} p$ data cover narrower range of $Q^{2}$ values and therefore the smallest $x_{\min }=0.002$ is rather large as far as GS is concerned. Nevertheless the results for $e^{-} p$ and $e^{+} p$ scattering restricted to the same kinematical region are very similar except for some small systematic shift of the exponent $\lambda$ towards higher values in the $e^{-} p$ case [31].

Natural question concerning other deep inelastic scattering experiments arises. Here we have $\mu p$ scattering experiments: EMC [37] with the smallest Bjorken $x_{\min }=0.0175$, BCMDS [38] $\left(x_{\min }=0.07\right), \operatorname{NMC}[39]\left(x_{\min }=0.008\right), \mathrm{E} 665[40]\left(x_{\min }=8 \times 10^{-4}\right)$ and SLAC $e^{-} p$ experiment [41] $\left(x_{\min }=0.063\right)$. Let us remind here that the lowest Bjorken $x$ for the combined HERA analysis is by far the smallest: $x_{\min }=5.52 \times 10^{-6}$. This alone singles out HERA data as the best experimental sample to look for geometrical scaling. Since, basing on our experience with $e^{-} p$ data, we expect some possible systematic differences due to the projectile used in different experiments, we have analyzed following the steps of section 2 - each of these experiments separately. We have observed, indeed, rather large systematic differences between various experiments in the overlapping region of Bjorken $x$ 's (i.e. for large $x$ ) as far as $\left\langle\lambda_{\min }\right\rangle$ and $\left\langle\left\langle\lambda_{\min }\right\rangle\right\rangle$ are concerned. However, due to large errors of $\left\langle\lambda_{\min }\right\rangle$ and $\left\langle\left\langle\lambda_{\min }\right\rangle\right\rangle$ it is difficult to quantify the amount of violation of GS in these experiments. As far as E665 data is concerned, we could see qualitative agreement with the combined HERA data, although E665 data suffer from very large errors for $\left\langle\lambda_{\min }\right\rangle$ and $\left\langle\left\langle\lambda_{\min }\right\rangle\right\rangle$. More detailed analysis and comparison of DIS experiments [37-41] with the combined HERA data [30] will be presented elsewhere.

It is worthwhile mentioning that our results for the best value of the exponent $\lambda$ are in good agreement with the quality factor analysis of ref. [26, 27] $\lambda=0.321 \pm 0.056$ and with later analysis of the combined HERA data only [29] $\lambda=0.31$. In both cases, however, the analysis was constrained to $x<0.01$ with some additional cuts on $Q^{2}$ and $y$ which have not been applied in our study.

Obviously our method can be applied to test different forms of scaling variable $\tau$ which follow from the QCD non-linear evolution equations [26-29]. Finally, the question of the possible existence of geometrical scaling for the charm cross-section $F_{2}^{c} / Q^{2}[28,42]$ where the charm quark mass plays an important role may also be studied with the method proposed in this paper.

\section{Acknowledgments}

MP would like to thank Robi Peschanski for discussion and for drawing his attention to the quality factor studies of geometrical scaling and Gosta Gustafson for pointing out the problem of the charm $F_{2}^{c}$ studied in ref. [42]. This work was supported by the Polish NCN grant 2011/01/B/ST2/00492.

Open Access. This article is distributed under the terms of the Creative Commons Attribution License which permits any use, distribution and reproduction in any medium, provided the original author(s) and source are credited. 


\section{References}

[1] A. Stasto, K.J. Golec-Biernat and J. Kwiecinski, Geometric scaling for the total $\gamma^{*} p$ cross-section in the low $x$ region, Phys. Rev. Lett. 86 (2001) 596 [hep-ph/0007192] [INSPIRE].

[2] A.H. Mueller, Parton saturation: an overview, hep-ph/0111244 [INSPIRE].

[3] L. McLerran, Strongly interacting matter matter at very high energy density: 3 lectures in Zakopane, Acta Phys. Polon. B 41 (2010) 2799 [arXiv:1011.3203] [InSPIRE].

[4] A. Freund, K. Rummukainen, H. Weigert and A. Schafer, Geometric scaling in inclusive eA reactions and nonlinear perturbative QCD, Phys. Rev. Lett. 90 (2003) 222002 [hep-ph/0210139] [INSPIRE].

[5] A. Freund, H. Weigert, A. Schafer and K. Rummukainen, Saturation from nonlinear $p Q C D$ at small $x$ in e $p$ and $e$ A processes, Acta Phys. Polon. B 33 (2002) 3057 [InSPIRE].

[6] N. Armesto, C.A. Salgado and U.A. Wiedemann, Relating high-energy lepton-hadron, proton-nucleus and nucleus-nucleus collisions through geometric scaling, Phys. Rev. Lett. 94 (2005) 022002 [hep-ph/0407018] [INSPIRE].

[7] C. Marquet and L. Schoeffel, Geometric scaling in diffractive deep inelastic scattering, Phys. Lett. B 639 (2006) 471 [hep-ph/0606079] [INSPIRE].

[8] L. McLerran and M. Praszalowicz, Saturation and scaling of multiplicity, mean $p_{\mathrm{T}}, p_{\mathrm{T}}$ distributions from $200 \mathrm{GeV}<\sqrt{s} 7 \mathrm{TeV}$, Acta Phys. Polon. B 41 (2010) 1917 [arXiv: 1006.4293] [INSPIRE].

[9] L. McLerran and M. Praszalowicz, Saturation and scaling of multiplicity, mean $p_{T}$ and $p_{T}$ distributions from $200 \mathrm{GeV}<\sqrt{s}<7 \mathrm{TeV}$ - addendum, Acta Phys. Polon. B 42 (2011) 99 [arXiv:1011.3403] [INSPIRE].

[10] M. Praszalowicz, Improved geometrical scaling at the LHC, Phys. Rev. Lett. 106 (2011) 142002 [arXiv: 1101.0585] [INSPIRE].

[11] M. Praszalowicz, Geometrical scaling in hadronic collisions, Acta Phys. Polon. B 42 (2011) 1557 [arXiv: 1104.1777] [INSPIRE].

[12] M. Praszalowicz, New look at geometrical scaling, arXiv:1112.0997 [INSPIRE].

[13] M. Praszalowicz, Geometrical scaling in high energy hadronic collisions, arXiv:1205.4538 [INSPIRE].

[14] L. Gribov, E. Levin and M. Ryskin, Semihard processes in QCD, Phys. Rept. 100 (1983) 1 [INSPIRE].

[15] A.H. Mueller and J.-W. Qiu, Gluon recombination and shadowing at small values of $x$, Nucl. Phys. B 268 (1986) 427 [INSPIRE].

[16] L.D. McLerran and R. Venugopalan, Computing quark and gluon distribution functions for very large nuclei, Phys. Rev. D 49 (1994) 2233 [hep-ph/9309289] [InSPIRE].

[17] L.D. McLerran and R. Venugopalan, Gluon distribution functions for very large nuclei at small transverse momentum, Phys. Rev. D 49 (1994) 3352 [hep-ph/9311205] [INSPIRE].

[18] L.D. McLerran and R. Venugopalan, Green's functions in the color field of a large nucleus, Phys. Rev. D 50 (1994) 2225 [hep-ph/9402335] [INSPIRE]. 
[19] A. Ayala, J. Jalilian-Marian, L.D. McLerran and R. Venugopalan, Quantum corrections to the Weizsacker-Williams gluon distribution function at small x, Phys. Rev. D 53 (1996) 458 [hep-ph/9508302] [INSPIRE].

[20] J. Kwiecinski and A. Stasto, Geometric scaling and QCD evolution, Phys. Rev. D 66 (2002) 014013 [hep-ph/0203030] [INSPIRE].

[21] J. Kwiecinski and A. Stasto, Large geometric scaling and QCD evolution, Acta Phys. Polon. B 33 (2002) 3439 [INSPIRE].

[22] E. Iancu, K. Itakura and L. McLerran, Geometric scaling above the saturation scale, Nucl. Phys. A 708 (2002) 327 [hep-ph/0203137] [INSPIRE].

[23] F. Caola and S. Forte, Geometric scaling from GLAP evolution, Phys. Rev. Lett. 101 (2008) 022001 [arXiv: 0802 .1878] [INSPIRE].

[24] K.J. Golec-Biernat and M. Wusthoff, Saturation effects in deep inelastic scattering at low $Q^{2}$ and its implications on diffraction, Phys. Rev. D 59 (1998) 014017 [hep-ph/9807513] [INSPIRE].

[25] K.J. Golec-Biernat and M. Wusthoff, Saturation in diffractive deep inelastic scattering, Phys. Rev. D 60 (1999) 114023 [hep-ph/9903358] [INSPIRE].

[26] F. Gelis, R.B. Peschanski, G. Soyez and L. Schoeffel, Systematics of geometric scaling, Phys. Lett. B 647 (2007) 376 [hep-ph/0610435] [INSPIRE].

[27] G. Beuf, R. Peschanski, C. Royon and D. Salek, Systematic analysis of scaling properties in deep inelastic scattering, Phys. Rev. D 78 (2008) 074004 [arXiv:0803.2186] [INSPIRE].

[28] G. Beuf, C. Royon and D. Salek, Geometric scaling of $F_{2}$ and $F_{2}^{c}$ in data and QCD parametrisations, arXiv:0810.5082 [INSPIRE].

[29] C. Royon and R. Peschanski, Studies of scaling properties in deep inelastic scattering, PoS (DIS 2010) 282 [arXiv: 1008.0261] [INSPIRE].

[30] H1 and ZEUS collaboration, F. Aaron et al., Combined measurement and QCD analysis of the inclusive $e^{ \pm} p$ scattering cross sections at HERA, JHEP 01 (2010) 109 [arXiv:0911.0884] [INSPIRE].

[31] T. Stebel, Quantitative analysis of geometrical scaling in deep inelastic scattering, arXiv: 1210.1567 [INSPIRE].

[32] H1 collaboration, C. Adloff et al., Deep inelastic inclusive ep scattering at low $x$ and a determination of $\alpha_{s}$, Eur. Phys. J. C 21 (2001) 33 [hep-ex/0012053] [INSPIRE].

[33] ZEUS collaboration, S. Chekanov et al., Measurement of the neutral current cross-section and $F_{2}$ structure function for deep inelastic $e+p$ scattering at HERA, Eur. Phys. J. C 21 (2001) 443 [hep-ex/0105090] [INSPIRE].

[34] J. Bartels, K.J. Golec-Biernat and H. Kowalski, A modification of the saturation model: DGLAP evolution, Phys. Rev. D 66 (2002) 014001 [hep-ph/0203258] [INSPIRE].

[35] H. Kowalski, L. Lipatov, D. Ross and G. Watt, Using HERA data to determine the infrared behaviour of the BFKL amplitude, Eur. Phys. J. C 70 (2010) 983 [arXiv:1005.0355] [INSPIRE].

[36] F. Caola, S. Forte and J. Rojo, HERA data and DGLAP evolution: theory and phenomenology, Nucl. Phys. A 854 (2011) 32 [arXiv:1007.5405] [InSPIRE]. 
[37] European Muon collaboration, J. Aubert et al., A detailed study of the proton structure functions in deep inelastic muon-proton scattering, Nucl. Phys. B 259 (1985) 189 [INSPIRE].

[38] BCDMS collaboration, A. Benvenuti et al., A high statistics measurement of the proton structure functions $F_{2}\left(x, Q^{2}\right)$ and $R$ from deep inelastic muon scattering at high $Q^{2}$, Phys. Lett. B 223 (1989) 485 [INSPIRE].

[39] New Muon collaboration, M. Arneodo et al., Measurement of the proton and deuteron structure functions, $F_{2}^{(p)}$ and $F_{2}^{(d)}$ and of the ratio $\sigma_{L} / \sigma_{T}$, Nucl. Phys. B 483 (1997) 3 [hep-ph/9610231] [INSPIRE].

[40] E665 collaboration, M. Adams et al., Proton and deuteron structure functions in muon scattering at $470 \mathrm{GeV}$, Phys. Rev. D 54 (1996) 3006 [InSPIRE].

[41] L. Whitlow, E. Riordan, S. Dasu, S. Rock and A. Bodek, Precise measurements of the proton and deuteron structure functions from a global analysis of the SLAC deep inelastic electron scattering cross-sections, Phys. Lett. B 282 (1992) 475 [INSPIRE].

[42] E. Avsar and G. Gustafson, Geometric scaling and QCD dynamics in DIS, JHEP 04 (2007) 067 [hep-ph/0702087] [INSPIRE]. 